\title{
Association between MTHFR gene polymorphisms (C677T, A1298C) and genetic susceptibility to prostate cancer: a meta-analysis
}

\author{
P.L. Chen ${ }^{1 *}$, W.T. Li ${ }^{2 *}$, J. Wang ${ }^{3 *}$, Y.D. Jiang ${ }^{1}$, P. Wu ${ }^{1}$, T. Chen ${ }^{1}$ and S.B. Zheng ${ }^{1}$ \\ 1'Department of Urology, Nanfang Hospital, Southern Medical University, \\ Guangdong Province, China \\ 2Department of Endocrinology, \\ The Third Affiliated Hospital of Southern Medical University, \\ Guangdong Province, China \\ ${ }^{3}$ Department of Radiation Oncology, Nanfang Hospital, Southern Medical University, \\ Guangdong Province, China
}

*These authors contributed equally to this study.

Corresponding author: S.B. Zheng

E-mail: Iwfineday@126.com

Genet. Mol. Res. 14 (4): 19191-19202 (2015)

Received August 4, 2015

Accepted October 29, 2015

Published December 29, 2015

DOI http://dx.doi.org/10.4238/2015.December.29.29

ABSTRACT. Genetic polymorphisms (C677T and A1298C) in methylenetetrahydrofolate reductase (MTHFR) were shown to be related to prostate cancer risk in previous studies; however, the results are controversial. We performed a meta-analysis of previous studies and quantitatively estimated these associations. Pubmed, Embase, and Cochrane Library Database were searched for published case-control studies evaluating the association between C677T (or A1298C) and prostate cancer risk. Pooled associations were presented as odds ratios (ORs) along with their 95\% confidence intervals. Twenty-one case control studies were identified for meta-analysis that included 21,581 participants. 
No significant associations were found between the MTHFR polymorphisms C677T or A1298C and prostate cancer risk in our meta-analysis. However, in subgroup analyses, the C677T CT polymorphism was associated with increased prostate cancer risk in East Asians (CT vs CC+TT: OR $=1.324, \mathrm{P}=0.03)$. The A1298C CC polymorphism in MTHFR was also linked to slightly reduced prostate cancer risk in European residents (CC vs $\mathrm{AC}+\mathrm{AA}: \mathrm{OR}=0.751, \mathrm{P}=0.004 ; \mathrm{CC}$ vs $\mathrm{AA}: \mathrm{OR}=0.768, \mathrm{P}=0.011$ ), whereas it was associated with a significantly increased prostate cancer risk in Asian residents ( $C C$ vs AA: OR $=1.862, P=0.006)$. The C677T CT polymorphism of MTHFR may be a risk factor for prostate cancer in East Asians. The association between the MTHFR A1298C CC genotype and prostate cancer risk may vary within different populations. Large-scale well-designed studies are required to confirm these associations.

Key words: A1298C; C677T; Methylenetetrahydrofolate reductase gene; Meta-analysis; Polymorphism; Prostate cancer

\section{INTRODUCTION}

Prostate cancer has been recognized as a common malignancy in males and is the sixth most common cause of cancer-related mortality (Ferlay et al., 2010). Although the etiology of prostate cancer remains largely unknown, it has been widely accepted that genetic factors play important roles in its pathogenesis.

DNA hypermethylation stimulates early oncogenesis, and DNA methylation status has been implicated in cancer development (Jones, 2014). Methylenetetrahydrofolate reductase (MTHFR) acts as a universal methyl group donor during DNA methylation and nucleotide synthesis. Thus, MTHFR deficiency may lead to hypomethylation, which is thought to confer a reduced risk of cancer development (Jacob et al., 1998). It has also been reported that hypomethylation of the CpG island inside the promoter of the glutathione-S-transferase P1 gene (GSTP1, a tumor suppressor gene) may activate its transcription, thus exerting protective effects against prostate cancer (Lee et al., 1997; Maldonado et al., 2014). Previous studies have explored the associations between MTHFR gene polymorphisms and prostate cancer susceptibility, although the findings have been controversial. A recently published meta-analysis that included 7000 cases revealed that the C677T polymorphism may confer a protective effect against prostate cancer in East Asians (Zhang et al., 2012). No significant associations between the risk of prostate cancer and the A1298C CC polymorphism in MTHFR have been observed in previous meta-analyses ( $\mathrm{Li}$ et al., 2012; Li and $\mathrm{Xu}, 2012$ ). However, the studies examined mostly included Caucasian participants rather than a diverse population that included East Asians as well as failed to include the study by Collin et al. (2009), which included 1562 cases and 2832 controls, and thus showed a relatively higher risk of bias. Recent studies suggested that African-Americans had higher prostate cancer risk and poorer prognosis compared to Caucasians, while East Asians had a lower risk of prostate cancer than did Caucasians (Ferlay et al., 2010; Lichtensztajn et al., 2013; Ito, 2014). This suggests that genetic factors may have different impacts on the risk of prostate cancer in different populations. Several recent studies (Fard-Esfahani et al., 2012; Kobayashi et al., 2012; Mandal et al., 2012; de Vogel et al., 2013; Jackson et al., 2013; López-Cortés et al., 2013; Ghasemi et al., 2014) investigated the 
association between MTHFR gene polymorphisms and prostate cancer risks, but found conflicting results. Thus, the impact of MTHFR gene polymorphisms (C677T and A1298C) on prostate cancer risk remains unclear.

In the current study, we conducted a meta-analysis of all available case-control studies of the C677T and A1298C polymorphisms in the MTHFR gene in order to more precisely and comprehensively estimate the associations between the MTHFR polymorphisms and prostate cancer risk.

\section{MATERIAL AND METHODS}

\section{Search strategy}

We conducted a systematic search of studies in the following databases: PubMed, Embase, and Cochrane library published until October 17, 2014 using the following search terms: "methylenetetrahydrofolate reductase", "prostate", and "polymorphism", with no further restrictions. Study titles and abstracts identified by searching were screened and the full-text of all potentially relevant studies was obtained. Retrieval and screening was conducted by 2 independent reviewers. In cases of duplicate publications, studies with the largest sample size or latest edition were selected. This study was performed according to the proposal of Meta-analysis of Observational Studies in Epidemiology group (Stroup et al., 2000).

\section{Selection criteria}

Studies were included in the current meta-analysis if they fulfilled the following criteria: 1) case-control studies evaluating the association between the C677T or A1298C polymorphisms and human prostate cancer risk, 2) diagnosis of prostate cancer confirmed by medical history interview or pathological examination, 3 ) odds ratio (OR) with $95 \%$ confidence interval $(\mathrm{Cl})$ for the association between the polymorphism and human prostate cancer risk was available or could be estimated, and 4) results were derived from suitable statistical methods. Studies were excluded if they met one of the followings: 1) publication type was a review, conference abstract, case report, editorial, letter, or meta-analysis, 2) OR with $95 \% \mathrm{Cl}$ or the genotype frequency for the association between polymorphism and human prostate cancer risk was unavailable or could not be estimated, and 3) insufficient information for data extraction.

\section{Data extraction}

Data extracted from eligible studies included the: name of the first author, publication year, country, region (Asia, America, or Europe), populations studied (Caucasians, East Asians, or mixed), genotyping methods [polymerase chain reaction-restriction fragment length polymorphism (PCR-RFLP), TaqMan, or others], control origin [publication base (PB), hospital base, and family base], sample sources (blood or specimen), diagnostic criteria for prostate cancer, total number of cases and controls included, numbers of genotypes in case and control groups, and Hardy-Weinberg equilibrium (HWE) in controls. Studies which included $>90 \%$ Caucasians were considered as studies in Caucasians. Studies with no clear description of ethnicity or studies with $<90 \%$ Caucasians were defined as "mixed". Two independent reviewers reviewed the eligible 
articles and extracted the above-mentioned information. Reviewers checked the data interactively, and reached agreement on the extracted information through discussion. If any discordance still existed, a specialist was consulted to make a final decision.

\section{Statistical analysis}

The association between the gene polymorphisms and prostate cancer risk (ORs and $95 \% \mathrm{Cls}$ ) was estimated for each study. The association was assessed using the allele model, codominant model, additive model, dominant model, and recessive model. HWE in controls was calculated using a goodness-of-fit chi-square test, which was available online (Rodriguez et al., 2009). Between-study heterogeneity was assessed using the $\chi^{2}$-based Q-test and $I^{2}$ statistics. Results of the $Q$ test, $P>0.1$, and $I^{2}<50 \%$ suggested homogeneity between studies. A fixed-effect model (Mantel-Haenszel) was used to estimate the pooled ORs. Otherwise, the random-effect model (DerSimonian and Laird) was applied. In addition to comparisons for total subjects, studies were categorized into different subgroups according to region (Asia, America, or Europe), populations (Caucasians, East Asians, or mixed), different genotyping methods used (PCR-RFLP, TaqMan, or other methods), and HWE (yes or no). Inverted funnel plots, the Begg test, and the the Egger test were used to examine publication bias for each polymorphism (linear regression analysis). The Duval and Tweedie non-parametric "trim and fill" method was used if publication bias existed $(P<$ 0.05 for Begg test or Egger test). Sensitivity analysis was performed by sequentially omitting single studies. Further, meta-cumulative analyses were performed in the order of ascending publication year or sample size to reveal the impact of a single study on the pooled OR.

The data were statistically analyzed using the Stata 12.0 software (StataCorp. LP, College Station, TX, USA) by 2 independent reviewers. Results were determined when the 2 analysts reached a consensus.

\section{RESULTS}

\section{Study characteristics}

The search and selection process for case-control studies are shown in Figure 1. Briefly, 72 articles were identified after the initial database search and exclusion of duplicates. After screening the titles and abstracts, 22 papers were excluded because 23 studies were not relevant to the study aims and 6 were reviews or meta-analyses. Twenty-one studies evaluating the potential impact of MTHFR polymorphisms on prostate cancer risk were retrieved. Two articles were included by manually assessing the references for the 21 full-text articles (Heijmans et al., 2003; Stevens et al., 2008). Moreover, 2 studies were excluded because they did not provide the exact genotypes and the OR $(95 \% \mathrm{Cl})$ for the association between gene polymorphisms and prostate cancer risk could not be estimated (Zacho et al., 2011; Jackson et al., 2013). As a result, 21 case-control studies were included in our meta-analysis (Kimura et al., 2000; Heijmans et al., 2003; Cicek et al., 2004; Singal et al., 2004; Van Guelpen et al., 2006; Johansson et al., 2007; Reljic et al., 2007; Marchal et al., 2008; Stevens et al., 2008; Collin et al., 2009; Muslumanoglu et al., 2009; Cai et al., 2010; Safarinejad et al., 2010; Wu et al., 2010; Küçükhüseyin et al., 2011; Fard-Esfahani et al., 2012; Kobayashi et al., 2012; Mandal et al., 2012; de Vogel et al., 2013; López-Cortés et al., 2013; Ghasemi et al., 2014). 


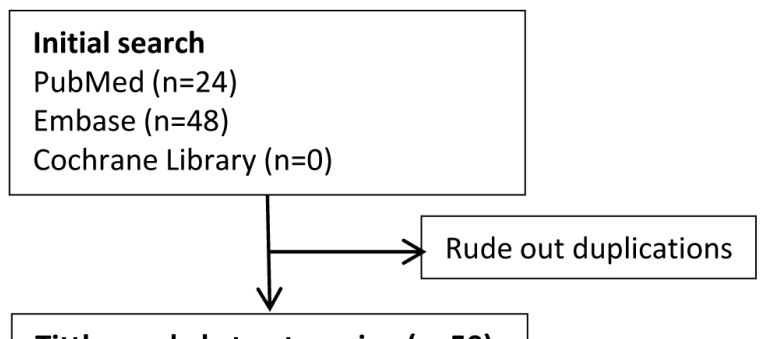

Tittles and abstracts review $(n=50)$

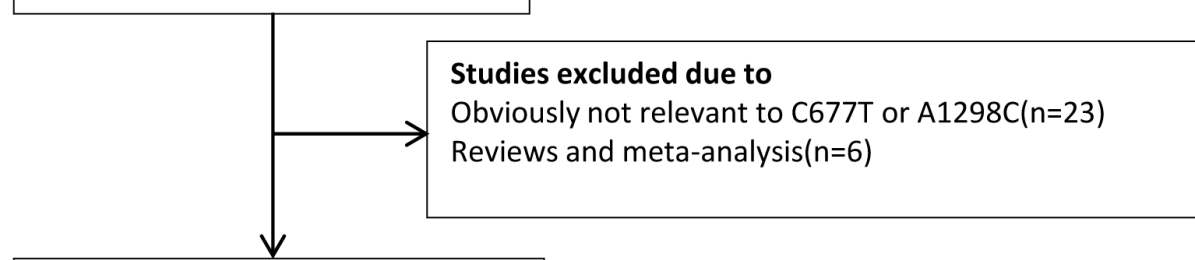

\section{Full-text and references} $\operatorname{review}(\mathbf{n}=21)$

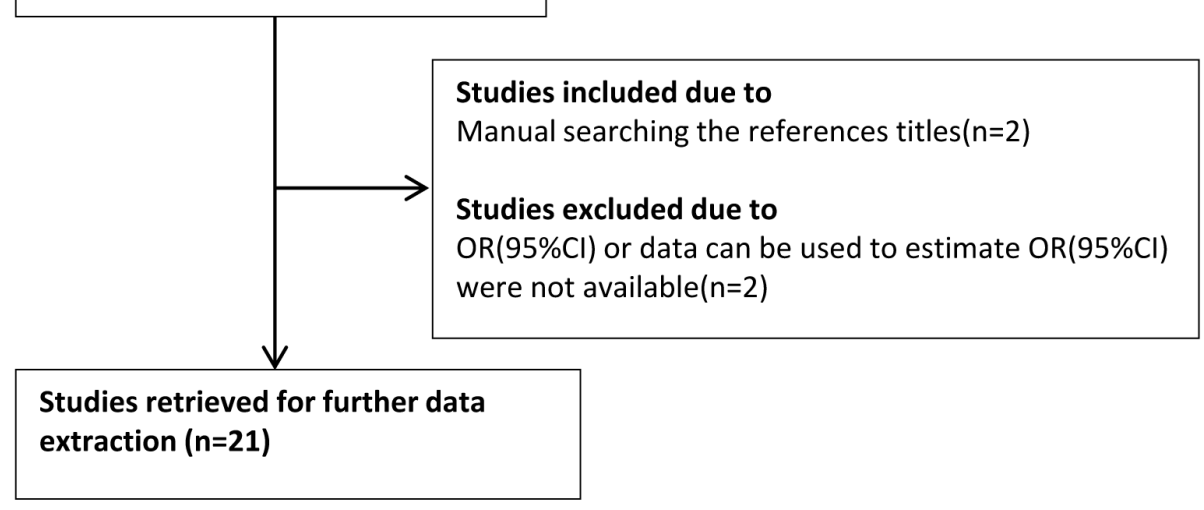

Figure 1. Flow chart of meta-analysis to identify associations between MTHFR gene polymorphisms (C677T, A1298C) and prostate cancer risk.

The main characteristics of the included studies are summarized in Table 1. All 21 studies investigated the impact of the C677T polymorphism on prostate cancer risk, while 10 also studied the impact of the A1298C polymorphism. A total of 10,529 cases and 11,052 controls were available for the estimation of C677T polymorphism, while 4255 cases and 6071 controls were included in the A1298T polymorphism analysis. Among all studies, 17 were conducted mostly in Caucasians (>90\%), 2 were conducted in East Asian populations, and 2 were in other populations (1 in Ecuadorians with no clearly described ethnicity, 1 in African-Caribbean population). For the A1298T polymorphism, 8 were performed in Caucasians, 2 in East Asians, and 1 in Ecuadorians. Genotyping methods used in the studies include PCR-RFLP and TaqMan, as well as other methods, such as amplification-refractory mutation system-PCR and matrix-assisted laser desorption/ ionization-time-of-flight mass spectrometry. 


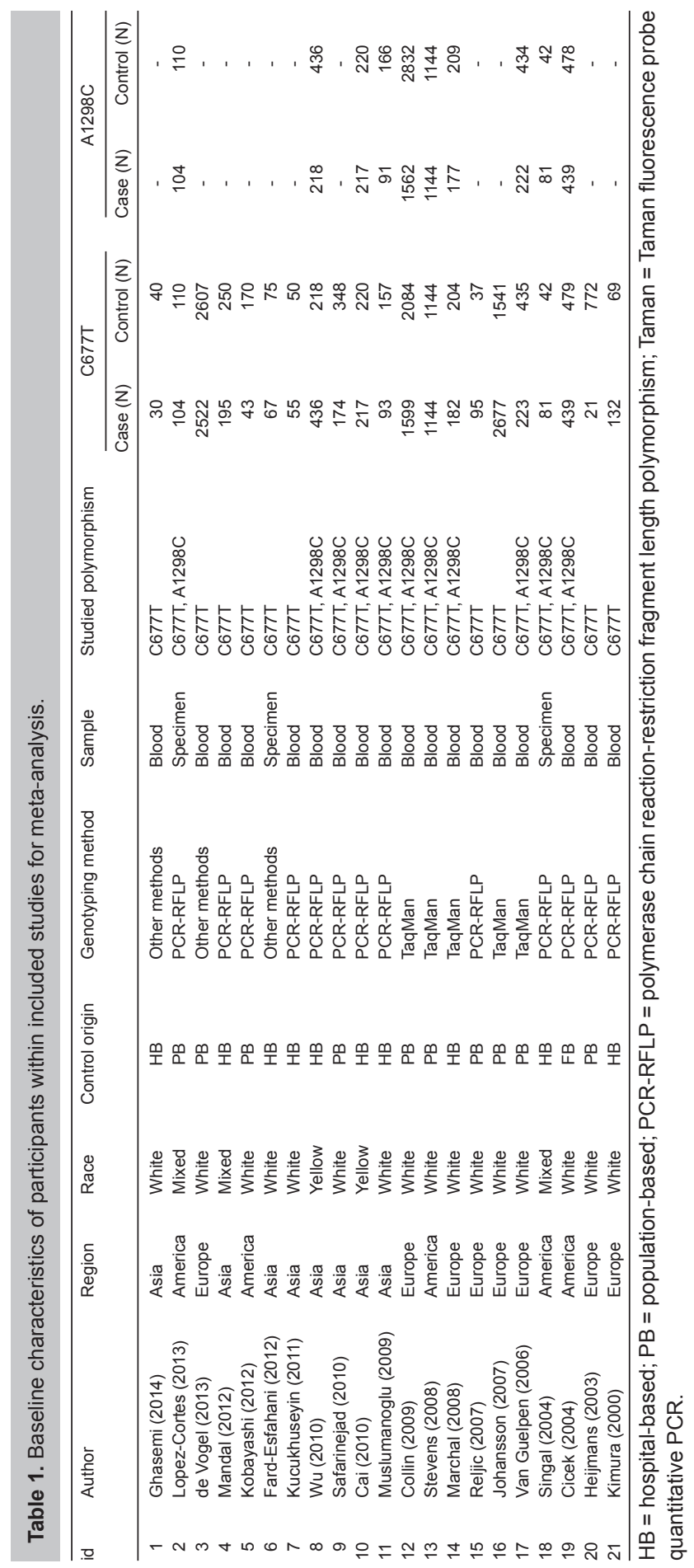




\section{Meta-analysis results}

For the C677T polymorphism, the main results of the meta-analysis are summarized in Table 2. Overall, no significant association was observed between C677T and prostate cancer risk based on meta-analysis using all genetic models. Subgroup analysis of studies of East Asians revealed deteriorative effects when using the co-dominant model (CT vs CC+TT: OR $=1.324$, $95 \% \mathrm{Cl}=1.028-1.706, \mathrm{P}=0.03$ ). Interestingly, subgroup analyses according to genotyping methods (allele, co-dominant, additive, and dominant models) revealed a significantly lower risk of prostate cancer in participants with the C677T polymorphism ( $\mathrm{T}$ vs C: OR $=0.867,95 \% \mathrm{Cl}=0.797-0.942$, $\mathrm{P}=0.001 ; \mathrm{CT}$ vs CC+TT: $\mathrm{OR}=0.869,95 \% \mathrm{Cl}=0.776-0.974, \mathrm{P}=0.016 ;$ TT vs $\mathrm{CC}: \mathrm{OR}=0.82$, $95 \% \mathrm{Cl}=0.691-0.973, \mathrm{P}=0.023 ; \mathrm{TT}+\mathrm{CT}$ vs $\mathrm{CC}: \mathrm{OR}=0.833,95 \% \mathrm{Cl}=0.747-0.928, \mathrm{P}=0.001)$. In these studies, control subjects did not reach HWE, while the results of meta-analysis suggested a protective role for the C677T polymorphism in prostate cancer in an additive model (TT vs CC: OR $=0.83,95 \% \mathrm{Cl}=0.708-0.973, \mathrm{P}=0.022$ ) . No significant associations were detected in subgroup analyses by region, control origin, and sample origin.

\begin{tabular}{|c|c|c|c|c|c|c|c|}
\hline & \multirow[t]{2}{*}{ Contrasting model } & \multirow[t]{2}{*}{ OR } & \multirow[t]{2}{*}{$95 \% \mathrm{Cl}$} & \multirow[t]{2}{*}{$\mathrm{P}$} & \multicolumn{2}{|c|}{ Heterogeneity } & \multirow[t]{2}{*}{ Model } \\
\hline & & & & & $P$ of $Q$-test & $1^{2}$ & \\
\hline \multicolumn{8}{|l|}{ Grouped by race } \\
\hline East Asian $(\mathrm{N}=2)$ & $\mathrm{CT}$ vs $\mathrm{CC}+\mathrm{TT}$ & 1.324 & $1.028-1.706$ & 0.03 & 0.197 & 38.4 & Fixed \\
\hline \multicolumn{8}{|c|}{ Grouped by genotyping methods } \\
\hline \multirow[t]{4}{*}{ Other methods $(\mathrm{N}=3)$} & T vs C & 0.867 & $0.797-0.942$ & 0.001 & 0.722 & 0 & Fixed \\
\hline & $\mathrm{CT}$ vs $\mathrm{CC}+\mathrm{TT}$ & 0.869 & $0.776-0.974$ & 0.016 & 0.908 & 0 & Fixed \\
\hline & TT vs CC & 0.82 & $0.691-0.973$ & 0.023 & 0.465 & 0 & Fixed \\
\hline & $\mathrm{TT}+\mathrm{CT}$ vs $\mathrm{CC}$ & 0.833 & $0.747-0.928$ & 0.001 & 0.837 & 0 & Fixed \\
\hline \multicolumn{8}{|l|}{ Grouped by HWE } \\
\hline HWE-N $(\mathrm{N}=4)$ & $\mathrm{TT}$ vs $\mathrm{CT}+\mathrm{CC}$ & 0.83 & $0.708-0.973$ & 0.022 & 0.233 & 28.3 & Fixed \\
\hline
\end{tabular}

T vs C: allele model; CT vs CC+TT: codominant model; TT vs CC: additive model; TT+CT vs CC: dominant model; TT vs $\mathrm{CT}+\mathrm{CC}$ : recessive model.

For the A1298C polymorphism, the main findings of the meta-analysis are summarized in Table 3. Similarly to findings from the C677T polymorphism, meta-analysis did not reveal a significant association between $\mathrm{A} 1298 \mathrm{C}$ and the risk of prostate cancer. However, subgroup analysis by study region implicated a protective effect of $\mathrm{A} 1298 \mathrm{C}$ against prostate cancer in European residents $(\mathrm{CC}$ vs $\mathrm{AC}+\mathrm{AA}$ : $\mathrm{OR}=0.751,95 \% \mathrm{Cl}=0.617-0.914, \mathrm{P}=0.004 ; \mathrm{CC}$ vs $\mathrm{AA}$ : $\mathrm{OR}=0.768,95 \% \mathrm{Cl}=0.627-0.942, \mathrm{P}=0.011)$. In Asian residents, we observed a significant deteriorative effect observed using a recessive model $(\mathrm{CC}$ vs $\mathrm{AA}$ : $\mathrm{OR}=1.862,95 \% \mathrm{Cl}=1.19$ 2.913, $\mathrm{P}=0.006$ ). No significant associations were detected in Caucasians and East Asians. In mixed populations, a significant protective effect against prostate cancer was observed when using the allelic, recessive, and additive models $(C$ vs $A$ : $O R=0.419,95 \% C l=0.253-0.696, P=0.001$; $\mathrm{CC}$ vs $\mathrm{AC}+\mathrm{AA}: \mathrm{OR}=5.022,95 \% \mathrm{Cl}=2.133-11.827, \mathrm{P}<0.001 ; \mathrm{CC}$ vs $\mathrm{AA}: \mathrm{OR}=3.508,95 \% \mathrm{Cl}=$ 1.402-8.776, $P=0.007$ ). However, population based-studies (PB subgroup) and studies conducted using the TaqMan PCR genotyping methods (the TaqMan subgroup) both revealed a protective role in recessive and additive models (for $\mathrm{PB}, \mathrm{CC}$ vs $\mathrm{AC}+\mathrm{AA}$ : $\mathrm{OR}=0.813,95 \% \mathrm{Cl}=0.692-0.956, \mathrm{P}$ $=0.012 ; \mathrm{CC}$ vs AA: OR $=0.825,95 \% \mathrm{Cl}=0.697-0.977, \mathrm{P}=0.026$; for TaqMan, CC vs AC+AA: OR $=0.814,95 \% \mathrm{Cl}=0.698-0.97, \mathrm{P}=0.011 ; \mathrm{CC}$ vs $\mathrm{AA}: \mathrm{OR}=0.823,95 \% \mathrm{Cl}=0.698-0.97, \mathrm{P}=0.02$ ). 
The PCR-RFLP subgroup analysis using the additive model also revealed that the presence of the $\mathrm{A} 1298 \mathrm{C}$ polymorphism was related to a higher risk of prostate cancer (CC vs AA: OR $=1.582$, $95 \% \mathrm{Cl}=1.17-2.141, \mathrm{P}=0.046)$, which was not consistent with results when using the allelic model (C vs A: OR $=0.739,95 \% \mathrm{Cl}=0.55-0.994, \mathrm{P}=0.046)$. Studies using pathological specimens as a genotyping sample source (specimen subgroup) revealed deteriorative effects using recessive and additive models (CC vs AC+AA: $\mathrm{OR}=5.022,95 \% \mathrm{Cl}=1.133-11.817, \mathrm{P} \leq 0.001 ; \mathrm{CC}$ vs AA: OR $=3.508,95 \% \mathrm{Cl}=1.402-8.776, \mathrm{P}=0.007)$. No other significant associations were detected in our meta-analysis as grouped by HWE.

\begin{tabular}{|c|c|c|c|c|c|c|c|}
\hline & \multirow[t]{2}{*}{ Contrasting model } & \multirow[t]{2}{*}{ OR } & \multirow[t]{2}{*}{$95 \% \mathrm{Cl}$} & \multirow[b]{2}{*}{$P$} & \multicolumn{2}{|c|}{ Heterogeneity } & \multirow[t]{2}{*}{ Model } \\
\hline & & & & & P of Q-test & $\mathrm{I}^{2}$ & \\
\hline \multicolumn{8}{|l|}{ Grouped by region } \\
\hline Asia $(\mathrm{N}=3)$ & $\mathrm{CC}$ vs $\mathrm{AA}$ & 1.862 & $1.190-2.913$ & 0.006 & 0.236 & 30.7 & Fixed \\
\hline \multirow[t]{2}{*}{ Europe $(\mathrm{N}=3)$} & $\mathrm{CC}$ vs $\mathrm{AA}$ & 0.768 & $0.627-0.942$ & 0.011 & 0.536 & 0.0 & Fixed \\
\hline & $\mathrm{CC} v s \mathrm{AC}+\mathrm{AA}$ & 0.751 & $0.617-0.914$ & 0.004 & 0.458 & 0.0 & Fixed \\
\hline \multicolumn{8}{|l|}{ Grouped by race } \\
\hline \multirow[t]{3}{*}{ Mixed $(\mathrm{N}=2)$} & C vs A & 0.419 & $0.253-0.696$ & 0.001 & 0.875 & 0.0 & Fixed \\
\hline & $\mathrm{CC}$ vs $\mathrm{AA}$ & 3.508 & $1.402-8.776$ & 0.007 & 0.67 & 0.0 & Fixed \\
\hline & $\mathrm{CC}$ vs $\mathrm{AC}+\mathrm{AA}$ & 5.022 & $2.133-11.827$ & $<0.001$ & 0.46 & 0.0 & Fixed \\
\hline \multicolumn{8}{|c|}{ Grouped by control origin } \\
\hline \multirow{2}{*}{$\mathrm{PB}(\mathrm{N}=5)$} & $\mathrm{CC}$ vs $\mathrm{AA}$ & 0.825 & $0.697-0.977$ & 0.026 & 0.373 & 3.9 & Fixed \\
\hline & $C C$ vs $A C+A A$ & 0.813 & $0.692-0.956$ & 0.012 & 0.258 & 25.6 & Fixed \\
\hline \multicolumn{8}{|c|}{ Grouped by genotyping methods } \\
\hline \multirow[t]{2}{*}{ PCR-RFLP $(\mathrm{N}=7)$} & C vs A & 0.739 & $0.550-0.994$ & 0.046 & 0.002 & 73.3 & Random \\
\hline & $\mathrm{CC}$ vs $\mathrm{AA}$ & 1.582 & $1.170-2.141$ & 0.003 & 0.099 & 46.0 & Fixed \\
\hline \multirow[t]{2}{*}{ TaqMan $(\mathrm{N}=4)$} & $\mathrm{CC}$ vs AA & 0.823 & $0.698-0.97$ & 0.02 & 0.472 & 0.0 & Fixed \\
\hline & $\mathrm{CC}$ vs $\mathrm{AC}+\mathrm{AA}$ & 0.814 & $0.695-0.954$ & 0.011 & 0.32 & 14.5 & Fixed \\
\hline \multicolumn{8}{|c|}{ Grouped by DNA source } \\
\hline \multirow[t]{3}{*}{ Specimen $(N=2)$} & C vs A & 0.419 & $0.253-0.696$ & 0.001 & 0.875 & 0.0 & Fixed \\
\hline & CC vs. AA & 3.508 & $1.402-8.776$ & 0.007 & 0.67 & 0.0 & Fixed \\
\hline & $\mathrm{CC}$ vs $\mathrm{AC}+\mathrm{AA}$ & 5.022 & $2.133-11.827$ & $<0.001$ & 0.46 & 0.0 & Fixed \\
\hline
\end{tabular}

C vs A: allele model; $A C$ vs AA+CC: codominant model; $C C$ vs AA: additive model; $C C+A C$ vs $C C$ : dominant model; $C C$ vs $A C+A A$ : recessive model.

\section{Sensitivity analysis}

Meta-cumulative analysis revealed that López-Cortés et al. (2013) and Ghasemi et al. (2014) observed a profound impact on the pooled ORs regarding the associations between the C677T polymorphism and prostate cancer risk. However, only López-Cortés et al. (2013) found a significant association between the $\mathrm{A} 1298 \mathrm{C}$ polymorphism and risk of prostate cancer. For the C677T polymorphism, a significant association only remained for the other genotyping methods subgroup and the subgroup in Hardy-Weinberg disequilibrium after excluding the 2 studies. When the results of Ghasemi et al. (2014) were excluded, the significance of the pooled ORs for the A1298C polymorphism was still robust under all contrast models. Omitting the other single study did not lead to a significant change in the pooled OR (data not shown).

\section{Publication bias}

The results of the Begg and Egger tests suggested the existence of publication bias within studies examining the A1298C polymorphism when using the allelic model (Begg test $\mathrm{P}=0.152$; 
Egger test: $P=0.043)$. However, no significant change in the $O R$ was detected after processing using the "trim and fill" methods. Neither the Begg test nor Egger test revealed significant publication bias in the remaining $\mathrm{A} 1298 \mathrm{C}$ polymorphism and all C677T polymorphism models.

\section{DISCUSSION}

MTHFR is a key enzyme involved in folate and vitamin B12 metabolism, homocysteine metabolism, and DNA synthesis. It is a critical catalyzer that converts 5,10-methylenetetrahydrofolate to a major form of serum folate, 5-methyltetrahydrofolate, which acts as the methyl donor in purine and thymidine synthesis. Because of its vital role in cellular processes, MTHFR and its C677T and A1298C polymorphisms have been associated with the pathogenesis of many different diseases such as cancer, including colorectal, gastric, cervical, and prostate cancers (Kimura et al., 2000; Haghighi et al., 2009; Neves Filho et al., 2010; Zhu et al., 2013). Currently, studies of prostate tumor cells have revealed that some pathways involved in folate metabolic disorders may contribute to the pathogenesis of prostate cancer, such as CpG islands and histone methylation, DNA uracil misincorporation, and chromosomal rearrangements (Collin, 2013). Studies also indicated that high serum vitamin B12 and folate levels may confer a higher prostate cancer risk (Collin et al., 2009). With every $5 \mathrm{nM}$ increase in serum folate levels, there was an increased risk of prostate cancer (risk ratio $=1.04 ; 95 \% \mathrm{Cl}=1.00-1.07 ; \mathrm{P}=0.042$ ) (Wang et al., 2014). Studies have indicated that the C677T or A1298C polymorphisms have a large impact on MTHFR activity, which may lead to altered serum folate levels (Collin et al., 2009). Although Safarinejad et al. (2010) found no difference of vitamin B12 and serum folate between the C677T or A1298C genotypes in Iranian, an Italian study revealed that for the C677T polymorphism, serum folate level was decreased in CC, CT, and TT subjects, and folate concentration was higher in CC than in AC or AA subjects for the A1298C polymorphism (Safarinejad et al., 2010; Zappacosta et al., 2014). Thus, there may be close associations between the C677T or A1298C polymorphisms of MTHFR and prostate cancer risk. However, whether these polymorphisms are associated with prostate cancer susceptibility remains unclear. In the current study, we performed an updated meta-analysis to comprehensively evaluate these associations.

Recent published meta-analyses regarding the association between the C677T polymorphism and prostate cancer risk have shown conflicting results. Li et al. (2012) suggested a slightly protective effect of C677T. However, Collin et al. (2009) and Zhang et al. (2012) found no association between C677T and prostate cancer risk. In contrast to previous meta-analyses, the present study included recently published studies, incorporating data form different populations and an article with more than 2000 Caucasian cases and 2000 controls. Although no significant association was found in the overall meta-analysis, subgroup analysis showed that the presence of C677T was associated with an increased prostate cancer risk in East Asians, supporting the results of Zhang et al. (2012).

Interestingly, we observed protective effects of the C677T polymorphism against prostate cancer in studies using other genotyping methods (amplification-refractory mutation system-PCR and matrix-assisted laser desorption/ionization-time-of-flight mass spectrometry). The significance of these findings remained robust even after omitting other articles in sensitivity analyses. These findings indicate that using unified genotyping methods is important for accurately assessing the association between the C677T polymorphism and prostate cancer risk.

For the A1298C polymorphism, previous published meta-analyses concluded that this polymorphism was not associated with prostate cancer risk, both in overall analysis and subgroup 
analysis by region, included populations, genotyping methods, or prostate cancer stages (Li et al., 2012; Li and Xu, 2012). However, previous meta-analyses only included 2723 patients and 3442 controls; these populations were much smaller compared to that used in our study. In the present study, 4255 cases and 6071 controls were included. We observed a significant cancer-inducing effect of the CC genotype in Asian residents and a slightly protective effect of the $\mathrm{C}$ allele and CC genotype against prostate cancer in European residents.

Furthermore, a significant cancer-inducing effect of the CC genotype was observed for the $\mathrm{A} 1298 \mathrm{C}$ polymorphism when DNA was extracted from specimens in additive and recessive models, whereas no significant associations were observed in studies examining blood DNA. These findings indicate that localized DNA mutations in prostate tissue are a more fundamental pathophysiological factor in cancer development rather than systematic mutation as reflected by the blood samples. In addition, the results of subgroup analysis suggested that the results of metaanalysis differed according to different study designs (PB or family base) and genotyping methods (PCR-RFLP or TaqMan), indicating that more precise designation and accurate approaches should be adopted in future studies.

There were several limitations to our study. First, some subgroups only included relatively small sample sizes, which may have led to reduced statistical power. Second, our study did not include results from genome-wide association studies, which may have affected the reliability of the results. Third, our study was based on unadjusted estimates and thus the impact of potential confounding could not be excluded. Fourth, multiple factors, such as geographic distribution, populations of the participants, study design, and methods used, may have contributed to heterogeneity among the included studies. Individual patient data-based meta-analysis may be helpful for clarifying their impact on the overall results.

In conclusion, our study provides an updated meta-analysis estimating the association between MTHFR gene polymorphisms and prostate cancer risk and included larger sample sizes compared to previous studies. The C677T polymorphism may be associated with an increased prostate cancer risk in East Asians. The presence of the A1298C polymorphism appears to be associated with an increased risk of prostate cancer in Asian residents, but a reduced risk in European residents. Large-scale well-designed studies are required to confirm the association between MTHFR polymorphisms and prostate cancer susceptibility in the future.

\section{Conflicts interest}

The authors declare no conflict of interest.

\section{ACKNOWLEDGMENTS}

We thank Medjaden Bioscience Limited for assisting in the preparation of this manuscript.

\section{REFERENCES}

Cai D, Ning L, Pan C, Liu X, et al. (2010). Association of polymorphisms in folate metabolic genes and prostate cancer risk: a case-control study in a Chinese population. J. Genet. 89: 263-267.

Cicek MS, Nock NL, Li L, Conti DV, et al. (2004). Relationship between methylenetetrahydrofolate reductase C677T and A1298C genotypes and haplotypes and prostate cancer risk and aggressiveness. Cancer Epidemiol. Biomarkers Prev. 13: $1331-1336$. 
Collin SM (2013). Folate and B12 in prostate cancer. Adv. Clin. Chem. 60: 1-63.

Collin SM, Metcalfe C, Zuccolo L, Lewis SJ, et al. (2009). Association of folate-pathway gene polymorphisms with the risk of prostate cancer: a population-based nested case-control study, systematic review, and meta-analysis. Cancer Epidemiol. Biomarkers Prev. 18: 2528-2539.

de Vogel S, Meyer K, Fredriksen A, Ulvik A, et al. (2013). Serum folate and vitamin B12 concentrations in relation to prostate cancer risk - a Norwegian population-based nested case-control study of 3000 cases and 3000 controls within the JANUS cohort. Int. J. Epidemiol. 42: 201-210.

Fard-Esfahani P, Mohammadi Torbati P, Hashemi Z, Fayaz S, et al. (2012). Analysis of relation between C677T genotype in MTHFR gene and prostatic cancer in Iranian males. Acta Med. Iran. 50: 657-663.

Ferlay J, Shin HR, Bray F, Forman D, et al. (2010). Estimates of worldwide burden of cancer in 2008: GLOBOCAN 2008. Int. J. Cancer 127: 2893-2917.

Ghasemi S, Tavakoli A, Moghadam M, Zargar MA, et al. (2014). Risk of prostate cancer and thrombosis-related factor polymorphisms. Biomed. Rep. 2: 53-56.

Haghighi MM, Radpour R, Mahmoudi T, Mohebbi SR, et al. (2009). Association between MTHFR polymorphism (C677T) with nonfamilial colorectal cancer. Oncol. Res. 18: 57-63.

Heijmans BT, Boer JM, Suchiman HE, Cornelisse CJ, et al. (2003). A common variant of the methylenetetrahydrofolate reductase gene (1p36) is associated with an increased risk of cancer. Cancer Res. 63: 1249-1253.

Ito K (2014). Prostate cancer in Asian men. Nat. Rev. Urol. 11: 197-212.

Jackson MD, Tulloch-Reid MK, McFarlane-Anderson N, Watson A, et al. (2013). Complex interaction between serum folate levels and genetic polymorphisms in folate pathway genes: biomarkers of prostate cancer aggressiveness. Genes Nutr. 8: 199-207.

Jacob RA, Gretz DM, Taylor PC, James SJ, et al. (1998). Moderate folate depletion increases plasma homocysteine and decreases lymphocyte DNA methylation in postmenopausal women. J. Nutr. 128: 1204-1212.

Johansson M, Van Guelpen B, Hultdin J, Wiklund F, et al. (2007). The MTHFR 677C --> T polymorphism and risk of prostate cancer: results from the CAPS study. Cancer Causes Control 18: 1169-1174.

Jones PA (2014). At the tipping point for epigenetic therapies in cancer. J. Clin. Invest. 124: 14-16.

Kimura F, Franke KH, Steinhoff C, Golka K, et al. (2000). Methyl group metabolism gene polymorphisms and susceptibility to prostatic carcinoma. Prostate 45: 225-231.

Kobayashi LC, Limburg H, Miao Q, Woolcott C, etal. (2012). Folate intake, alcohol consumption, and the methylenetetrahydrofolate reductase (MTHFR) C677T gene polymorphism: influence on prostate cancer risk and interactions. Front. Oncol. 2: 100.

Küçükhüseyin Ö, Kurnaz Ö, Akadam-Teker AB, Narter F, et al. (2011). Effects of the MTHFR C677T polymorphism on prostate specific antigen and prostate cancer. Asian Pac. J. Cancer Prev. 12: 2275-2278.

Lee WH, Isaacs WB, Bova GS and Nelson WG (1997). CG island methylation changes near the GSTP1 gene in prostatic carcinoma cells detected using the polymerase chain reaction: a new prostate cancer biomarker. Cancer Epidemiol. Biomarkers Prev. 6: 443-450.

Li D, Tian T, Guo C, Ren J, et al. (2012). No association of the MTHFR gene A1298C polymorphism with the risk of prostate cancer: a meta-analysis. Exp. Ther. Med. 3: 493-498.

Li XL and Xu JH (2012). MTHFR polymorphism and the risk of prostate cancer: a meta-analysis of case-control studies. Prostate Cancer Prostatic Dis. 15: 244-249.

Lichtensztajn DY, Gomez SL, Sieh W, Chung BI, et al. (2014). Prostate cancer risk profiles of Asian-American men: disentangling the effects of immigration status and race/ethnicity. J. Urol. 191: 952-956.

López-Cortés A, Jaramillo-Koupermann G, Muñoz MJ, Cabrera A, et al. (2013). Genetic polymorphisms in MTHFR (C677T, A1298C), MTR (A2756G) and MTRR (A66G) genes associated with pathological characteristics of prostate cancer in the Ecuadorian population. Am. J. Med. Sci. 346: 447-454.

Maldonado L, Brait M, Loyo M, Sullenberger L, et al. (2014). GSTP1 promoter methylation is associated with recurrence in early stage prostate cancer. J. Urol. 192: 1542-1548.

Mandal RK, Nissar K and Mittal RD (2012). Genetic variants in metabolizing genes NQO1, NQO2, MTHFR and risk of prostate cancer: a study from North India. Mol. Biol. Rep. 39: 11145-11152.

Marchal C, Redondo M, Reyes-Engel A, Perea-Milla E, et al. (2008). Association between polymorphisms of folate-metabolizing enzymes and risk of prostate cancer. Eur. J. Surg. Oncol. 34: 805-810.

Muslumanoglu MH, Tepeli E, Demir S, Uludag A, et al. (2009). The analysis of the relationship between A1298C and C677T polymorphisms of the MTHFR gene with prostate cancer in Eskisehir population. Genet. Test. Mol. Biomarkers 13: 641-645.

Neves Filho EH, Alves MK, Lima VP and Rabenhorst SH (2010). MTHFR C677T polymorphism and differential methylation status in gastric cancer: an association with Helicobacter pylori infection. Virchows Arch. 457: 627-633.

Reljic A, Simundic AM, Topic E, Nikolac N, et al. (2007). The methylenetetrahydrofolate reductase (MTHFR) C677T polymorphism and cancer risk: the Croatian case-control study. Clin. Biochem. 40: 981-985. 
Rodriguez S, Gaunt TR and Day IN (2009). Hardy-Weinberg equilibrium testing of biological ascertainment for Mendelian randomization studies. Am. J. Epidemiol. 169: 505-514.

Safarinejad MR, Shafiei N and Safarinejad S (2010). Relationship between three polymorphisms of methylenetetrahydrofolate reductase (MTHFR C677T, A1298C, and G1793A) gene and risk of prostate cancer: a case-control study. Prostate 70: 1645-1657.

Singal R, Ferdinand L, Das PM, Reis IM, et al. (2004). Polymorphisms in the methylenetetrahydrofolate reductase gene and prostate cancer risk. Int. J. Oncol. 25: 1465-1471.

Stevens VL, Rodriguez C, Sun J, Talbot JT, et al. (2008). No association of single nucleotide polymorphisms in one-carbon metabolism genes with prostate cancer risk. Cancer Epidemiol. Biomarkers Prev. 17: 3612-3614.

Stroup DF, Berlin JA, Morton SC, Olkin I, et al. (2000). Meta-analysis of observational studies in epidemiology: a proposal for reporting. Meta-analysis Of Observational Studies in Epidemiology (MOOSE) group. JAMA 283: 2008-2012.

Van Guelpen BR, Wirén SM, Bergh AR, Hallmans G, et al. (2006). Polymorphisms of methylenetetrahydrofolate reductase and the risk of prostate cancer: a nested case-control study. Eur. J. Cancer Prev. 15: 46-50.

Wang R, Zheng Y, Huang JY, Zhang AQ, et al. (2014). Folate intake, serum folate levels, and prostate cancer risk: a metaanalysis of prospective studies. BMC Public Health 14: 1326.

Wu HC, Chang CH, Tsai RY, Lin CH, et al. (2010). Significant association of methylenetetrahydrofolate reductase single nucleotide polymorphisms with prostate cancer susceptibility in Taiwan. Anticancer Res. 30: 3573-3577.

Zacho J, Yazdanyar S, Bojesen SE, Tybjaerg-Hansen A, et al. (2011). Hyperhomocysteinemia, methylenetetrahydrofolate reductase c.677C $>\mathrm{T}$ polymorphism and risk of cancer: cross-sectional and prospective studies and meta-analyses of 75,000 cases and 93,000 controls. Int. J. Cancer 128: 644-652.

Zappacosta B, Graziano M, Persichilli S, Di Castelnuovo A, et al. (2014). 5,10-Methylenetetrahydrofolate reductase (MTHFR) C677T and A1298C polymorphisms: genotype frequency and association with homocysteine and folate levels in middlesouthern Italian adults. Cell Biochem. Funct. 32: 1-4.

Zhang WB, Zhang JH, Pan ZQ, Yang QS, et al. (2012). The MTHFR C677T polymorphism and prostate cancer risk: new findings from a meta-analysis of 7306 cases and 8062 controls. Asian Pac. J. Cancer Prev. 13: 2597-2604.

Zhu J, Wu L, Kohlmeier M, Ye F, et al. (2013). Association between MTHFR C677T, MTHFR A1298C and MS A2756G polymorphisms and risk of cervical intraepithelial neoplasia II/III and cervical cancer: a meta-analysis. Mol. Med. Rep. 8: 919-927. 